\title{
Matemáticas y computación: Uso de programación visual para el desarrollo de material didáctico en un entorno educativo
}

\author{
Mathematics and computation: Using visual programming to develop didactic materials in a learning environment
}

\author{
Pablo C. Herrera \\ Universidad Peruana de Ciencias Aplicadas - \\ UPC, Perú \\ pablo@espaciosdigitales.org
}

\begin{abstract}
We analyse the problem of creating didactic material for teaching and evaluating mathematics in the first year of a School of Architecture. By using visual programming, science professor used codes (formulae) to represent in a software their proposals, instead of drawing them themselves. Through this experience we create a database of codes with computational solutions that allows faculty to modify, reuse, visualise and print in the same platform that she students will use while developing their designs. In this way we aim to maximise the link between mathematics and design as fundamental base for the control of complex shapes.
\end{abstract}

Keywords: Visual Programming, Mathematics Education, Architectural Education, Latin America, 3D Printing

\section{Introducción}

Desde la primera década del siglo XXI, se evidenció el uso de técnicas computacionales para explorar la complejidad de la forma y espacio usando las matemáticas. Investigaciones desarrolladas con fines pedagógicos (Pottmann et al. 2007; Woodbury, 2010; Choma, 2015) como el análisis de la práctica arquitectónica (Burry, 2010; Legendre, 2011) permiten entender que son experiencias particulares de una tendencia que se ve limitada por las razones que se analizan en esta investigación.

Aún así, este no es un tema del siglo XXI. Varios aspectos entre matemática $y$ arquitectura fueron investigados $y$ se documentaron sistemáticamente desde finales del siglo XX. Ese trabajo fue seleccionado por Williams y Ostwald (2015), y muestran en dos compendios, los momentos claves del uso de las matemáticas en la historia, enseñanza y teoría de la arquitectura. Esta selección, tomó como base más de 500 artículos publicados entre 1996 y el 2014 de las conferencias de Nexus: Architecture and Mathematics.

En general, los resultados de estas investigaciones son ejemplos concretos de cómo las matemáticas han contribuido en la arquitectura, especialmente en su historia, deconstruyendo metódicamente las emblemáticas construcciones de cada época.

\section{Matemáticas e implementación}

Los factores que dificultan la implementación sistemática de las matemáticas en la educación del arquitecto son tan variados, que las soluciones sostenibles a largo plazo son limitadas. Los problemas de implementación son los mismos que los encontrados en la implementación de programación (Herrera, 2013a; Herrera 2013b), en donde el aprendizaje por instrucciones se pierde, si no se dirige a problemáticas particulares de diseño.

Para el caso de los estudiantes de artes, Andersen et. al. (2003) afirman que "la mayoría de los estudiantes de artes liberales están más inclinados a temas de tipo abierto en el que el análisis, la discusión y la interpretación son las competencias básicas, y son menos propensos a tener interés en temas absolutos y cerrados como las matemáticas y la programación" (p. 109).

Tomando el caso de Latinoamérica, que es la región en donde se desarrolla este caso de estudio, se verifica que en las pruebas como PISA nuestros alumnos, antes de ingresar a la educación superior, están por debajo de los resultados mínimos en habilidades matemáticas. Rivas (2015) indica que "el 63\% de los alumnos evaluados no alcanzó el nivel II, que es considerado el mínimo para dominar conocimientos matemáticos fundamentales" (p. 190). En ese nivel, de un total de seis, los estudiantes no alcanzan a "elaborar y trabajar con modelos para situaciones complejas y trabajar estratégicamente utilizando capacidades de razonamiento y reflexión amplias y bien desarrolladas", es decir, "solamente pueden realizar las tareas mas fáciles y más obvias" (OECD, 2014, p. 4). Ello nos coloca en una situación difícil antes de 
empezar, por lo que brindar el mejor soporte tecnológico al área de Ciencias desde el campo de la arquitectura, en especial desde su área computacional, es la principal premisa de esta investigación.

Fortes y Marquez (2010, p. 1-2) sostienen tres limitaciones en la implementación de las matemáticas: la poca cantidad de horas asignadas a los diferentes cursos, limitando desarrollar ejercicios aplicados a la arquitectura; el número elevado de grupos de estudiantes y finalmente, el lenguaje específico y preciso que complica la enseñanza. La primera limitación ha sido superada en otras investigaciones, distribuyendo temas coincidentes con el área de comunicación o expresión, con el fin de no repetir contenidos, y dejar más horas en matemática que afiancen lo requerido. La segunda limitación ha sido superada con tutorías y prácticas dirigidas con el fin de complementar cualquier vacío dejado en clases numerosas. Sin embargo, con respecto al lenguaje, ha sido difícil encontrar una propuesta satisfactoria.

Ozcan y Akarun (2001) advirtieron que en la educación del diseñador, "los cursos de matemáticas ofrecidos en los primeros años de estudios, no son considerados como parte del desarrollo creativo, y se ven como cursos obligatorios y aburridos: una repetición monótona de temas de la secundaria" (p. 27).

También Kline (2012 [1967]) explica que para los estudiantes de humanidades "las matemáticas les parecen poco atrayentes y hasta sosas; pero adquieren un significado pleno cuando se les presentan en su contexto cultural" (p.17).

Aunque los profesores de matemáticas promueven la integración utilizando objetos arquitectónicos orientados a un contexto familiar, (como ocurre con la enseñanza de la programación), esta no sólo se logra con ejemplos que demuestren qué hacer con las matemáticas en un futuro, por cuanto si su integración es sólo referencial y no aplicada a un diseño, el estudiante en muchos casos no podrá asociar la abstracción de las matemáticas y lo concreto de la arquitectura. Es decir, el resolver problemas en primer año de estudios, sobre situaciones que ocurrirán en su práctica profesional futura, que será apoyada además por software especializado, producirá un efecto mínimo si no se asocian a problemas de diseño con los que normalmente el alumno se enfrentaría en un taller de diseño. Postura que también sostienen García y Albert (2005) al referirse de manera general que "los cursos de matemáticas no están desarrollados en el contexto de la arquitectura y el diseño" (p. 343), y que a partir de los contenidos tradicionales de la ingeniería, "han aportado poco a resolver la problemática del aprendizaje de las matemáticas" (p. 346).

De manera general, los ejercicios se orientan a la proporción y geometría, como es el caso peruano de Ugarte y Yucra (2011) en donde se espera que el alumno por si mismo relacione soluciones de ejercicios de matemática y su aplicación en la arquitectura.

\section{Del contenido al material didáctico}

Las razones por las que se aborda la problemática con el fin de elaborar material didáctico, específicamente la estandarización del mismo, es que los diferentes esfuerzos desde el punto de vista de los docentes no reflejan los resultados que ellos esperan. Por lo tanto, en vez de discutir los contenidos para la enseñanza de las matemáticas que es una tarea amplia y requiere una investigación transversal entre profesores de matemática y arquitectos, se buscó el espacio de trabajo con el fin de proponer un lenguaje común en donde se puedan explorar soluciones a problemas de matemática y de diseño, en vez de analizar en esta primera parte el contenido o temas.

Por ello, no se cuestionó, la relevancia ni pertinencia de los problemas propuestos, si no nos concentramos en enseñarles a resolver y explorar soluciones a sus problemas en la plataforma de trabajo planteada.

\section{Plataforma de trabajo propuesta}

La primera pregunta que les hice fue, ¿cómo pueden elaborar y proponer sus problemas sin dibujarlos?. A lo que contestaron: "es fácil, todo el resultado esta en nuestra imaginación, lo vemos simplemente". En conversación con los participantes, se encontró que toda esa habilidad matemática, se ve limitada, en algunos casos, al enfrentarse a una tecnología que no se adaptaba a la naturaleza de sus procesos. En este caso se trataba de docentes de Ciencias que aprendían a dibujar por computador, buscando una habilidad que no es una práctica habitual en matemáticas, con el fin de alcanzar la misma representación que está resuelta en sus mentes.

Es así que para el caso de estudio analizado, la experiencia previa para elaborar material didáctico se desarrollaba en diferentes plataformas informáticas. Algunos usaban programa de propietario como Microsoft Power Point, Paint y Microsoft Word o programas libres como Google SketchUp, Winplot, GeoGebra o InkScape. Con excepción de GeoGebra, todos los demás programas, fueron aprendidos de manera autoasistida. Del conjunto de estos programas, los profesores destacaron que las desventajas de los de representación, era la poca flexibilidad para controlar la escala, se invertía demasiado tiempo en la elaboración y dependían de las limitaciones del programa. Se les explicó que la única ventaja de utilizar programas como Google SketchUp o AutoCAD era la escala, por cuanto los mismos problemas del dibujo, los encontrarían alli.

Sin embargo, los profesores de matemáticas, encontraron ventajas en GeoGebra: les permitía utilizar el lenguaje de las matemáticas, sentían que ahorraban tiempo, trabajaban a escala o la edición era más rápida. Esta es la solución utilizada de manera general para la producción del material didáctico para visualizar y graficar como lo ha descrito Berenguer, et al. (2011). A pesar que permite exportar en STL, la edición en el programa destino es muy complicada. La imposibilidad de fabricar sus propuestas impresas en 3D o cortadas con laser, marcaba una limitación, por cuanto se buscaba la integración de toda la solución en una misma plataforma.

Así, se descartó como plataforma de trabajo un programa 
de representación, partiendo de las afirmaciones de Broadbent (1971) quien señala que "los dibujos, maquetas, descripciones por escrito, e incluso los programas de computador, todos "se hacen cargo" e imponen sus propias características a lo que se está diseñando" (p. 31); las de Herbert (1993) que, "cualquier dibujo se vuelve obsoleto casi instantáneamente por el siguiente dibujo" (p. 18) y; las de Gross, (1985) quien sostiene que "los dibujos son útiles para indicar soluciones de diseño específicas, pero no para indicar rangos de posibles decisiones. Por ejemplo, en un solo dibujo uno no puede fácilmente expresar un rango de composiciones de fachadas alternativas" (p. 6).

El efecto de utilizar un software interactivo tiene sus limitaciones cuando se trata de matemática y diseño. Como lo señala Turkle (1995), el software "toma la forma de una simulación de alguna realidad (...) donde el aprendizaje se basa en la exploración lúdica y no en un conjunto de reglas" (p. 60-61), como si sucede con las matemáticas. Es así que en muchos casos, la enseñanza de soluciones a un problema se asemeja a las prácticas de dibujo, repeticiones de un mismo procedimiento con el fin de alcanzar la habilidad por ensayo y error. Por otro lado, como puntualiza Legendre (2011) "para trabajar con matemáticas, no se debe utilizar una interfaz, por cuanto con ella no se puede determinar la autoridad del diseñador" (p. 17), dado que un programador desarrolló las soluciones previamente.

Se revisó sin embargo, que la exploración computacional entre matemática y en diseño no es un problema reciente. Pero en sus más de 40 años, sólo ha sido abordada desde la perspectiva del arquitecto o diseñador y hay muy pocos trabajos del area de ciencias dirigidos a experiencias en arquitectura.

Los casos en los que se utilizó programación escrita propuestas por arquitectos, son las experiencias de Montgomer y Boxerman (1968) usando FORTRAN; Mitchell et. al. (1988) usando PASCAL; Maeda (1999) usando DBN; Ozel (2004) usando VBA para AutoCAD; Baerlecken y Kobiella (2008) usando Rhinoscripting; y para el caso de la programación visual, Issa (2013 [2010]) con Grasshopper y Modelab (2015) con Dynamo.

En general, las experiencias señaladas se desarrollaron en talleres de diseño donde se encontró: Que no son entornos de aprendizaje inicial de las matemáticas como el caso de estudio que se presenta, por cuanto son utilizadas en su mayoría en la práctica profesional y; Que fueron autogestionarias desde la arquitetura, es decir, no fueron desarrolladas con otras áreas especializadas en matemáticas.

La programación escrita, tiene muchos referentes y guías como punto de partida para su enseñanza en arquitectura. Estos son los casos de plataformas como Processing (Reas y Fry, 2007, 2014; Terzidis, 2009; Alencar y Celani, 2014), Autolisp (Celani, 2003; Krawczyk, 2010) o Rhinoscripting. Pero este tipo de documentación, es habitualmente usada por estudiantes que están finalizando estudios de grado, maestría y doctorado. En el caso de los alumnos de los primeros años, la autoeducación es limitada a imitar y no a explorar, pero dado su aporte transversal a la enseñanza de la computación, serán tomados en cuenta para una propuesta de contenidos para una implementación a largo plazo.

En experiencias previas, se concluyó que la programación escrita tiene mucho potencial para la exploración de formas complejas, pero tiene también varias limitaciones en su implementación (Herrera, 2013a), por lo tanto, se eligió la programación visual como punto de partida.

Se propuso Grasshopper, que opera en Rhinoceros, por ser una plataforma abierta y usada en los cursos de la facultad de arquitectura donde se realizó el estudio. Ello permite acceder a laboratorios donde los profesores de matemáticas pueden desarrollar sus propuestas y posteriormente ser usada por estudiantes en los cursos de computación.

Se evaluó Dynamo, pero al igual que GeoGebra, la carencia de técnicas para la etapa de fabricación la dejó fuera de la implementación. Además, con la aparición de Dynamo Studio, las posibilidades de continuar siendo gratuita son limitadas. Sin embargo, el uso de Rhynamo permitirá integrar las soluciones entre Grasshopper con Dynamo y con ello, facilitar su conexión con Autodesk Revit, otro de los programas preferidos por los estudiantes. En ese mismo sentido, en el 2015, Graphisoft, propietaria de ArchiCAD, lanzó la conexión con Grasshopper, lo que abre otras posibilidades de aplicación para este proyecto y la integración de sus resultados con otras plataformas.

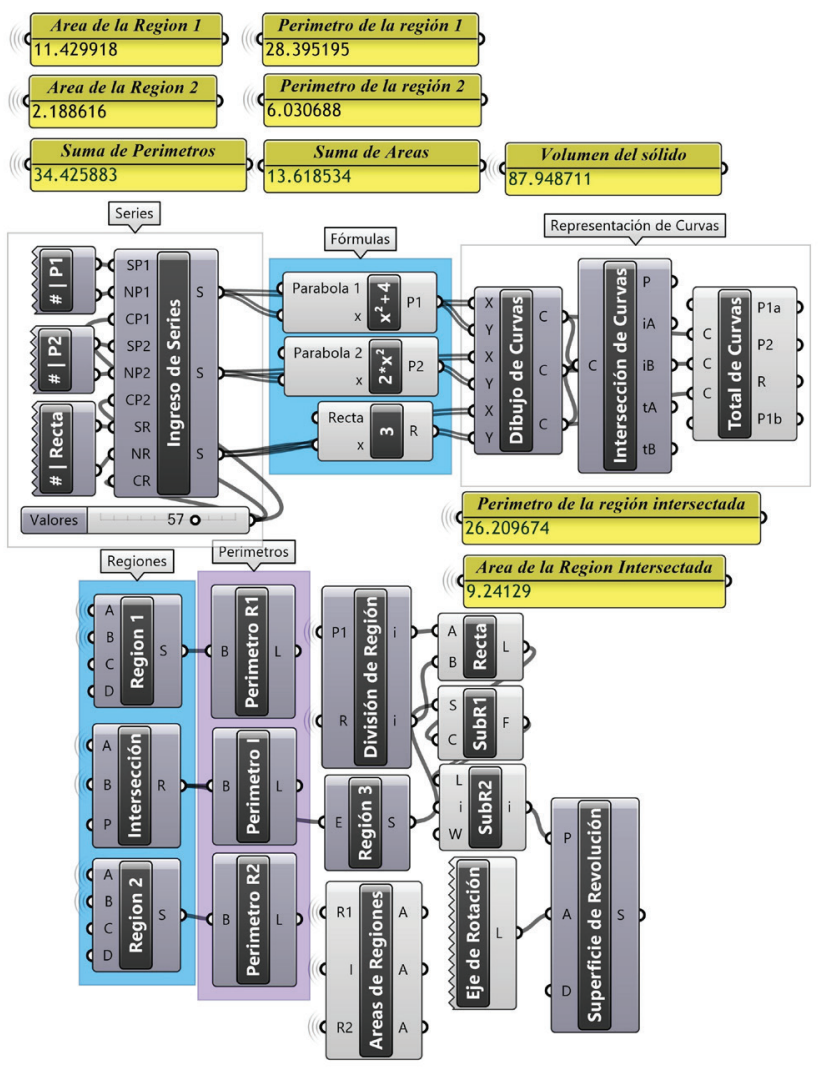

Figura 1: Diagrama que representa una intersección de dos parábolas y una recta girando en el eje x. Las variaciones en las Figuras 2 y 4. 
Como se mencionó, la premisa de fabricar no sólo debería permitir la impresión en tres dimensiones, sino dar la posibilidad de utilizar cartón o papel para armar modelos geométricos partiendo de un volumen utilizando laser de corte. Grasshopper, les permitió a los profesores realizar los desarrollos de figuras geométricas para elaborar el material en 2 y 3 dimensiones, como se puede ver en la Figura 2.

El ejemplo, es el resultado del uso de fórmulas para intersectar dos parábolasy una recta, donde la región resultante, revoluciona sobre un eje. La definición de este ejercicio se puede ver en la Figura 1., y el resultado de la variación de parámetros en la Figura 4.

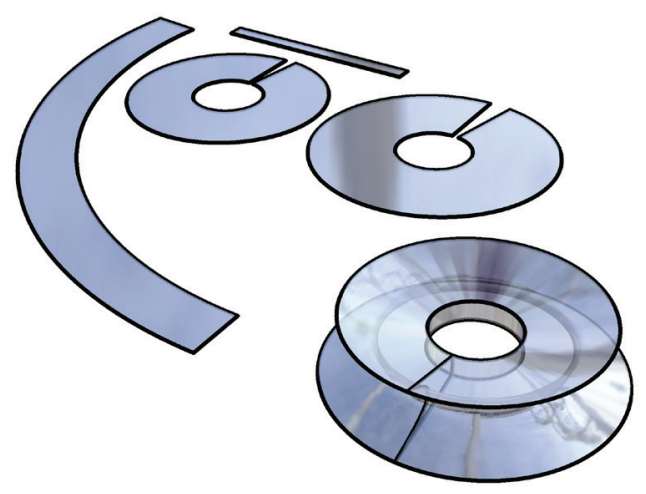

Figura 2: Volúmen y su desarrollo.

Así, esta investigación tuvo como objetivo general ofrecer una plataforma de trabajo a profesores de matemáticas en una facultad de arquitectura con el fin de desarrollar material didáctico, enfocado a la exploración de soluciones de problemas de ciencias.

Se propusieron cinco objetivos específicos: (1) establecer el mismoentorno de trabajo para la exploración de problemas de matemáticas y a la vez de diseño; (2) evidenciar que la visualización y fabricación de problemas de matemáticas, puede ser potenciada usando la programación; (3) evidenciar la exploración del proceso sobre la solución; (4) establecer que un repositorio de soluciones computacionales facilitará a los docentes la exploración de posibilidades en vez de dibujar cada vez sus propuestas y; (5) evidenciar que para acercar al estudiante a la exploración de formas y la fabricación de las mismas, la tecnología de impresión 3D es un recurso importante.

\section{Metodología}

El procedimiento inicial consistió en implementar un taller de programación visual dirigido a docentes de matemáticas del primer año de una facultad de arquitectura, tomando en cuenta que "el potencial de las matemáticas en la exploración de diseños, es mas variado e interesante en las primeras fases del proceso de lo que es en etapas posteriores" (Ozel, 2001, p. 196). Se documentó una expriencia previa con alumnos de arquitectura (Herrera, 2013b) de tercer año, y los resultados en la implementación de programación estaban distanciados del aprendizaje de matemáticas y de una verdadera solución a problemas de diseño. Por lo tanto, se espera que esta exploración en los primeros años, en donde también se familiarizan con problemas de diseño, sea la suficiente base para integrar computación, matemáticas y arquitectura con el fin de solucionar problemas particulares.

El taller fue solicitado por el área de Ciencias, con el fin de cubrir carencias en representación gráfica (su principal limitación), tomando en cuenta, que esta especialidad no tiene el dibujo como una exigencia en su aprendizaje. Se desarrolló material didáctico y exploraron las posibilidades de diseño con principios matemáticos (usando programación) y no con la manipulación directa de una geometría (programa interactivo). La metodología del taller partió de experiencias previas (Herrera, 2007, 2009, 2011, 2013b) en las que el pensamiento computacional se fortalecía por la experiencia de los participantes, en vez de las propuestas por el instructor del taller.

Se tomó esta premisa: si aprendían a programar usando sus procedimientos, la exploración de posibilidades permitiría crear y modificar más ejercicios, que sólo dibujarlos, con el fin de relacionarlos a formas arquitectónicas complejas.

\section{Caso de estudio}

El primer taller fue de 15 horas en 5 sesiones de 3 horas cada una. Dos semanas antes de iniciar, el autor seleccionó y resolvió algunos procedimientos de los cuadernos de trabajo del año 2015 de los cursos: Nivelación de Matemática, Matemática Básica I y II con énfasis en el Cálculo Diferencial e Integral. El objetivo era diseñar un conjunto de soluciones que evidencien que la plataforma propuesta reunía las condiciones y proponía la motivación para usarla de manera sostenible. Durante las sesiones, en un trabajo mutuo, se desarrollaron diagramas y definiciones en Grasshopper como el resultado de la Figura 3. 

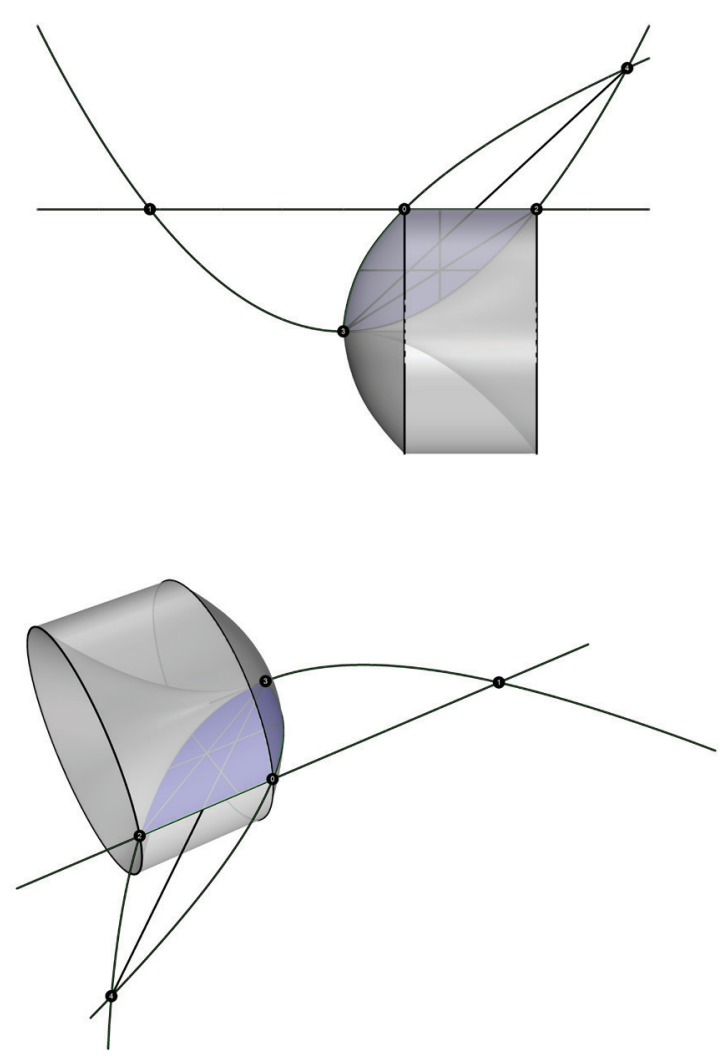

Figura 3: Intersección de dos parábolas y una recta.

En esa primera etapa iniciada el 2014, el número de participantes fue de 11, entre coordinadores de curso y profesores. Tuvo por finalidad familiarizar a los participantes con el proyecto. En ese sentido, los ejercicios fueron elegidos por el autor, quien a su vez, debía familiarizarse con los tipos de problema a resolver y de sus soluciones.

Por limitaciones administrativas, este proyecto se retrasó un año y se reanudó nuevamente en el 2015. En esta segunda etapa el número de profesores se limitó a coordinadores y fue un total de 8. Se establecieron sesiones de asesoría de 4 horas por semana divididas en dos días, limitados por la disponibilidad de laboratorios y la coincidencia de horarios entre los participantes. Se buscó que identificaran los problemas que más tiempo demandaban desarrollar y modificar. Los ejercicios no fueron elegidos por el autor, sino por los participantes. La condición, era que escogieran tipologías de ejercicios transversales a varios cursos. A partir de ellos, se empezó a elaborar un conjunto de definiciones con las soluciones a esos problemas.

En esta etapa, cada ejercicio era observado y criticado con el fin de tener todas las variables de control para que pueda ser modificado a futuro. Por ejemplo, si una forma, que utiliza lineas rectas, medio y cuarto de círculo podría tener otro parámetro que modifique su forma. Propusieron que los semicírculos puedan convertirse en trapecios. De tal forma, que la construcción de cada variación, era fortalecida por los participantes, quienes veían lo simple que era hacer
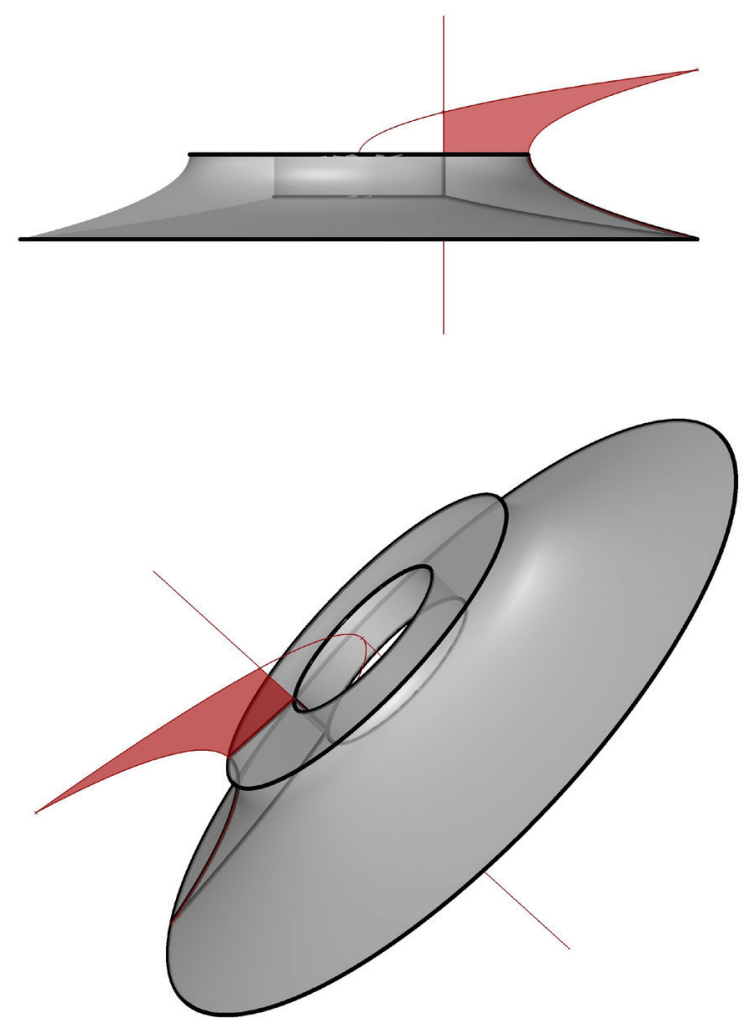

Figura 4: División de una región que revoluciona sobre un eje.

esos cambios cuando la definición contenía la estructura de solución al problema. Para cada problema, se elaboraba un listado de criterios a completar.

En paralelo a ambos procesos, se fue investigando las diferentes maneras en que otros programadores han resuelto problemas similiares utilizando Grasshopper, pero dada la particularidad de estos, casi todas las definiciones han sido únicas.

\section{Impresión 3D y matemáticas}

Slavkovsky (2012, p. 36) propuso imprimir en 3D material didáctico para enseñar geometría utilizando diferentes programas como Blender, HeksCAD, OpenSCAD, Google SetchUp, MeshLab, AdMesh, Mathematica y AutoCAD. Estas ofrecen la posibilidad de exportar a un programa de impresión 3D, pero todas son plataformas limitadas, tanto para producir código como para reusarlo. Unas con problemas relacionados con la escala, exactitud y otras con problemas de fabricación y documentación.

Dentro de todas las etapas y tomando como base la iniciativa de Knill y Slavkovsky (2013) que propusieron ilustrar las matemáticas utilizando impresoras 3D, se consideró recrear el avance de los resultados usando tecnología FDM, con el fin de explorar diferentes propuestas que pudieran ser usadas como material didáctico en futuras clases con estudiantes. Se utilizó una MakerBot Replicator de Quinta Generación, que es un estándar en los laboratorios de la facultad de arquitectura. 

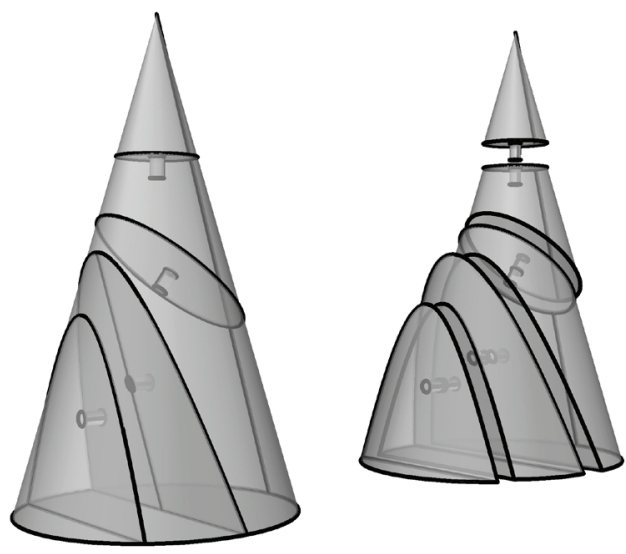

Figura 5: Cono de Apolonio modelado en Rhinoceros. A la izquierda rmado y a la derecha despiezado.

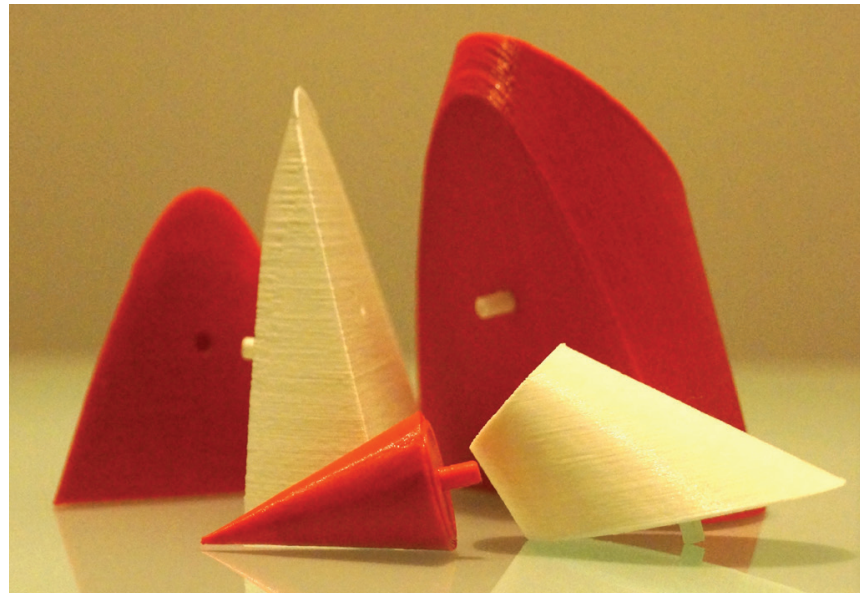

Figura 6 Impresión 3D de las 5 piezas que conforman el Cono de Apolonio, se utilizaron dos materiales para diferenciar las formas.

Lo particular de los casos desarrollados, hace que conseguir material didáctico en físico sea una limitante, especialmente si se requiere en una demanda mínima.

Por ejemplo, el caso de un Cono de Apolonio, que permite explicar cómo las secciones cónicas producen el círculo, la elipse, la parábola y la hipérbola, como en la Figura 5. Objeto que es por lo general encargado a artesanos y producido en madera bajo un largo proceso de trabajo, y que hoy junto a otros objetos se producirá en función de la demanda de lo que se requiera en los cursos. Así, gracias a la tecnología de impresión 3D se logran ejemplos como el de la Figura 6.

\section{Resultados Preliminares}

Durante estas primeras dos etapas de implementación, por primera vez, los profesores del área de Ciencias no se centraron en las particularidades del dibujo, ni en los problemas de fabricación de modelos o maquetas geométricas, sino en el desarrollo de sus fórmulas y procesos.

Asimismo, el diagrama y su proceso, que funcionan como una base de datos, les permitió decidir en qué momento podían obtener información del modelo, como perímetros, áreas, sumatorias de estas últimas, división de regiones, revoluciones, volúmenes, entre otro tipo de datos que no tenían limitaciones.

El impacto inmediato de esta experiencia, fue facilitar a los docentes una plataforma de trabajo que permita incorporar el pensamiento computacional en áreas donde las matemáticas tienen un proceso complejo de implementación.

El impacto a largo plazo es que este y los siguientes resultados se integren en el día a día de los estudiantes, luego de haber finalizado los cursos de matemáticas.

\section{Conclusiones}

El objetivo general fue cumplido de manera satisfactoria, los profesores encontraron una plataforma que coincide con sus requerimientos. Ese resultado confirma que el entorno de trabajo es el mismo para propósitos de diseño. Se evidenció asimismo, que la visualización y fabricación de problemas de matemáticas puede ser potenciada con la programación, profundizando el proceso, por cuanto el resultado siempre es una consecuencia de la modificación de parámetros. Finalmente, la impresión 3D, ha cumplido un rol fundamental en complementar la propuesta gráfica con la física.

$\mathrm{Si}$ al principio no se cuestionaron los tipos de problemas, la posibilidad de modificar sus propuestas plantea a los docentes el reto de buscar otras propuestas a los problemas existentes. Al superar la limitación del dibujo y ver la propuesta en tres dimensiones, surgieron inquietudes con el fin de proponer nuevas formas.

No se puede afirmar que el tipo de plataforma empleada sea la adecuada para todos los casos. Para el nuestro, dadas las condiciones de infraestructura y experiencia, se vuelve una implementación sostenible. Sin embargo, la metodología permitirá a otros investigadores explorar nuevas posibilidades con otras plataformas como Processing, Dynamo o Generative Components.

En función de los resultados, la cantidad de secciones y profesores en el área, en la siguiente etapa se definirá la elección de coordinadores por cada curso que lideren la implementación y uso de la base de datos, supervisadas por el autor. Este proceso llevará a seleccionar que tipo de habilidades se desea que alumno desarrolle para iniciar la construcción de dicha base de datos para ejercicios y evaluaciones. Este banco de datos, facilitará la modificación de los problemas propuestos, así como la representación de los ejercicios con un lenguage gráfico estandarizado. El banco de datos, permitirá que nuevos profesores tengan la posibilidad de revisar un histórico de ejercicios donde los parámetros serán el punto de partida para incorporar nuevas propuestas o integrarse a las existentes.

Luego de la puesta en marcha de la base de datos se espera que los profesores produzcan su primer libro con los resultados de esta experiencia. Material que junto a los ejercicios impresos 
en 3D, permitirá acercar las matemáticas a los alumnos. Para finales del 2015, se ha encargado la compra de cabezales de impresión Makerbot para nuestros equipos. Lo particular de esta adquisición, será que permitirá imprimir con apariencia de madera, piedra o cerámica, y acercará de esta manera la realidad de los objetos a una simulación casi perfecta.

Luego de este último proceso, se espera desarrollar un taller de soluciones computaciones en diseño con estudiantes que originalmente participaron de esta experiencia, con el fin de buscar el uso de las matemáticas para desarrollar sus propuestas.

Finalmente, tanto matemática como programación, convergen en lo que Papert (1980) propuso: si "hablamos matemáticas a una computadora podemos generalizarlo como la visión de un aprendizaje en el Mathland -donde las matemáticas pueden llegar a ser un vocabulario natural-" (p. 6 y 39) y eso es lo que ha permitido la programación a los profesores y se espera sea el canal de vínculo con los estudiantes.

La principal contribución de esta investigación, es que desde ciclos iniciales, los estudiantes conozcan la naturaleza de lo que nos exige un problema de forma y espacio en diseño, sin las limitaciones de la tecnología interpuesta para aprenderla e integremos de manera gradual procesos necesarios para el desarrollo de un pensamiento computacional cuando la complejidad del problema lo requiera.

\section{Agradecimientos}

A la Universidad Peruana de Ciencias Aplicadas por el fondo concursable otorgado para esta investigación y al área de Ciencias, por su disposición en ser parte de este proyecto. De manera especial agradecer el compromiso de cada uno de los profesores y coordinadores que son parte de esta iniciativa.

\section{Referencias}

Alencar, V., Celani, G. (2013). The Art of Computer Programming: Translating Pionner Programs. En XVII SIGraDi Conference Proceedings. pp. 500-504.

Andersen, P., Bennedsen, J., Brandorff, S., Caspersen, M., Mosegaard, J., (2003) Teaching Programming to Liberal Arts Students - A Narrative Media Approach. ACM SIGCSE, 35(3), 109-113.

Baerlecken, D., Kobiella, O. (2008). Math Objects. An Origin of Architecture. 26th. eCAADe Conference Proceedings, pp.677684.

Berenguer, M., Delgado, A., Fortes, M., Márquez, M., Pasadas, M., Rodríguez, M. (2011). Uso de Geogebra como complemento en la enseñanza de matemáticas en el Grado de Arquitectura. En IX Jornadas de Redes de Investigación en Docencia Universitaria.

Burry, J., Burry, M. (2010). The New Mathematics of Architecture. New York: Thames \& Hudson.

Celani, G. (2003). CAD Criativo. Recuperado de http://www. fec.unicamp.br/ celani/cad-criativo.htm

Choma, J. (2015). Morphing: A Guide to Mathematical
Transformation for Architects and Designers. New York: Laurence King Publishing.

Fortes, M., Márquez, M. (2010). Innovación Didáctica en la enseñanza de las Matemáticas en la titulación de arquitecto mediante el uso de las TIC. En II Congreso Internacional de Didácticas, pp. 1-6.

Garcia, M., Albert, J. (2005). Desarrollos matemáticos en arquitectura. En: Acta Latinoamericana de Matemática Educativa, 18, pp. 341-347.

Herrera, P. (2007). Solución de problemas relacionados al diseño de superficies complejas: Experiencia de programación en la educación del arquitecto. En XI SIGraDi Conference Proceedings. pp. 97-101.

Herrera, P. (2009). Patrones y convenciones en el uso de Rhinoscripting. En XIII SIGraDi Conference Proceedings. pp. 340-342.

Herrera, P. (2011). Rhinoscripting y Grasshopper a través de sus instructores: Un estudio de patrones y usos. En XV SIGraDi Conference Proceedings. pp. 180-183.

Herrera, P. (2013a). Patrones en la Enseñanza de la Programación en Arquitectura: De la Hetero-Educación a la Auto-Educación en Latinoamérica. En XVII SIGraDi Conference Proceedings. pp. 555-559.

Herrera, P. (2013b). Reutilizando códigos en arquitectura como mecanismos de información y conocimieno: De la programación Escrita a la Visual. En Rodriguez, D.; Tosello, M.E.; Sperling, D. (Eds.), Didactica proyectual y entornos postdigitales. Prácticas y reflexiones en escuelas latinoamericanas de Arquitectura y Diseño (pp. 238-253). Mar del Plata: Universidad Nacional de Mar del Plata.

Issa, R. (2013 [2010]). Essential Mathematics for Computational Design. Tercera Edición. http://www.rhino3d.com/download/ rhino/5.0/EssentialMathematicsThirdEdition/ Recuperado el 25 de noviembre de 2013.

Kline, M. (2012 [1967]). Matemáticas para los estudiantes de humanidades. México: Fondo de Cultura Económica.

Knill, O., Slavkovsky, E. (2013). Illustrating Mathematics using 3DPrinters.http://www.math.harvard.edu/ knill/3dprinter/ documents/trieste.pdf Recuperado el 18 de agosto de 2014.

Krawczyk, R. (2010). The Codewriting Workbook. Creating Computational Architecture in Autolisp. New Jersey: Princeton Architectural Press.

Legendre, G. (2011). Mathematics of Space. Architectural Design (Jul-Aug). Profile No. 212, 81(4).

Maeda, J. (1999). Design by Numbers. Cambridge: MIT Press.

Mitchell, W., Liggett, R., Kvan, T. (1987). The Art of Computer Graphics Programming. New York: Van Nostrand Reinhold.

ModeLab. The Dynamo Primer. First Edition V1.2. Recuperado de http://dynamoprimer.com/

Montgomer, R., Boxerman, S. (1968). An Applied Mathematics Course for Architects and Urban Designers. Journal of Architetural Education, 22(2/3), 29-31.

OECD Lo que los estudiantes saben y pueden hacer: Rendimiento de los estudiantes en matemáticas, lectura y ciencias. Recuperado de http://www.oecd.org/pisa/ 
keyfindings/PISA2012_Overview_ESP-FINAL.pdf

Ozcan, O., Akarun, L., (2001). Mathematics and Design Education. Design Issues. 17(3), 26-34.

Ozel, F. (2004), Modulation and Mathematics in Generative Building Design. En 22nd eCAADe Conference Proceedings, pp.195-200

Papert, S. (1980). Mindstorms: Children, Computers, and Powerful Ideas. New York: Basic Books.

Pottmann, H., Asperl, A., Hofer, M., Kilian, A., (2007). Architectural Geometry. Pennsylvania: Bentley Institute Press.

Reas, C., Fry, B. (2014), Processing. A Programming Handbook for Visual Designers and Artists. Cambridge: MIT Press.

Rivas, A. (2015). América Latina después de PISA: Lecciones aprendidas de la educación en siete países 2000-2015. Buenos Aires: Fundación CIPPEC.
Slavkovsky, E. (2012). Feasibility Study For Teaching Geomtry and Other Topics using Three-Dimensional Printers. A Thesis in the Field of Mathematics for Teaching. Cambridge: Harvard University.

Terzidis, K. (2009). Algorithms for Visual Design Using the Processing Language. New York: Wiley.

Turkle, S. (1995). Life on the Screen. Identity in the Age of the Internet. New York: Simon \& Chuster.

Ugarte, F., Yucra, J. (2014[2011]). Matemáticas para Arquitectos I. Lima: Facultad de Arquitectura, PUCP.

Williams, K., Ostwald, M. (2015). Architecture and Mathematics from Antiquity to the Future. Volumen I: Antiquity to the 1500s y Volumen II: The 1500s to the Future. New York: Springer.

Woodbury, R. (2010). Elements of Parametric Design. New York: Routledge. 